\title{
Slowhand: Does time perception change in peri-hand space?
}

\author{
Jacob S. Aday ${ }^{1}$ (D) $\cdot$ Christopher C. Davoli ${ }^{1} \cdot$ Emily K. Bloesch $^{1}$
}

Published online: 25 April 2019

(C) The Psychonomic Society, Inc. 2019

\begin{abstract}
A variety of attentional and perceptual changes occur in peri-hand space, including increases in visual temporal acuity. These changes in cognition have been related to an increase in magnocellular visual processing. Other magnocellular-related processes have been shown to enhance temporal sensitivity and lead to time overestimation. We hypothesized that a similar slowing of time perception would occur in peri-hand space. To this end, we had participants complete either a temporal bisection task or a verbal time estimation task with their hands near to or far from the test stimuli. Contrary to our predictions, we found no differences in time perception in peri-hand space. We situate our findings within the context of a contemporaneous study by Qi, Wang, He, and $\mathrm{Du}$ (2019), which produced conflicting results when using a temporal reproduction task to investigate the same phenomenon. The disparate results might relate to the fragility of peri-hand effects and/or to the tasks tapping into different aspects of time perception. Further research will be needed to fully elucidate the nuances of peri-hand space and temporal processing.
\end{abstract}

Keywords Peri-hand space $\cdot$ Time perception $\cdot$ Temporal processing $\cdot$ Temporal bisection task $\cdot$ Verbal estimation task

Our hands are of critical importance when interacting with the physical world. As such, researchers have found a variety of attentional and perceptual changes in peri-hand space, including faster target detection (Reed, Grubb, \& Steele, 2006), more thorough visual searches (Abrams, Davoli, Du, Knapp, $\&$ Paull, 2008), altered emotional processing (Davoli, 2010; Du, Wang, Abrams, \& Zhang, 2017), and enhanced visual perception (Schendel \& Robertson, 2004). Most pertinently, several studies have noted alterations in temporal processing in peri-hand space. When the hands are held near to the stimuli being assessed, performance is improved on tasks such as temporal fusion (Goodhew, Gozli, Ferber, \& Pratt, 2013) and temporal gap detection (Gozli, West, \& Pratt, 2012), which require high temporal sensitivity. Additionally, Thomas (2015) found that a power grasp, but not a precision grasp, improved performance in a task requiring high temporal acuity (e.g., a motion detection task). Stimuli in peri-hand space are thought to receive more attentional resources in order to facilitate action, leading to enhanced temporal

Jacob S. Aday

aday1js@cmich.edu

1 Department of Psychology, Central Michigan University, Mount Pleasant, MI, USA sensitivity (Goodhew et al., 2013). Although these studies suggest that temporal processing can be enhanced in perihand space, it is still unclear whether and how peri-hand space affects the subjective experience of time.

Changes to attention and perception in peri-hand space appear to be due at least in part to a biasing of information toward the magnocellular visual pathway (Abrams \& Weidler, 2014; Goodhew et al., 2013; Gozli et al., 2012), sometimes referred to as the "action" pathway. Relative to the parvocellular pathway, magnocellular neurons have high temporal sensitivity (i.e., high conduction speeds) and large receptive fields and process more coarse features of objects. The increased processing speed of visual information in the magnocellular pathway might underlie enhancements in visual temporal acuity. In support of the magnocellular account of peri-hand space, Abrams and Weidler found that cognitive changes in peri-hand space were eliminated when magnocellular neurons were suppressed.

It seems that when visual temporal acuity is altered, so, too, is the subjective perception of time. Emotion is one domain in which this relationship has been demonstrated: States of fear have been shown to enhance temporal sensitivity (Kobayashi \& Ichikawa, 2016) and have been linked with altered perception of time across a variety of paradigms (Droit-Volet \& Meck, 2007; Tipples, 2008; Stetson, Fiesta, \& Eagleman, 2007). Motion is another instance in which this relationship 
has been shown: Motion detection relies on heightened temporal acuity (Watson, 1986), and the perception of motion in static images leads to the experience of prolonged exposure to the images (Droit-Volet, Fayolle, Lamotte, \& Gil, 2013). Together, these studies support the notion that when temporal acuity is altered, the subjective perception of time, unsurprisingly, is distorted as well-suggesting that peri-hand space, which has been shown to alter temporal acuity (Goodhew et al., 2013; Gozli et al., 2012), may also affect time perception.

The prior literature suggests that there are several interconnections among temporal acuity, peri-hand space, and time perception. To review, temporal acuity has been shown to be enhanced in peri-hand space, and these changes are thought to rely in part on the magnocellular pathway. Other magnocellular-related processes have documented effects on temporal sensitivity and perception. Given these connections, we have reason to expect altered time perception when attending to stimuli near the hands.

To test the hypothesis that time perception will slow down in peri-hand space, we recruited participants to perform standard time perception tasks (either a temporal bisection task [Exp. 1] or a verbal estimation task [Exp. 2]) with their hands either near to or far from the stimuli. The two experiments were run concurrently, with participants being randomly assigned to one of the two tasks, in order to control for timeof-semester effects. In the bisection task, participants were first familiarized with a "short" and a "long" duration. They were then shown stimuli for durations between those two anchors and asked to judge which anchor the presented stimulus was closest to. In the verbal estimation task, participants were explicitly told the durations of two anchors (200 and 1,800 $\mathrm{ms}$ ) and then were shown stimuli for durations between those two anchors and asked to estimate in milliseconds the duration of the stimuli.

\section{Experiment 1}

In Experiment 1, participants completed a temporal bisection task, which yields subjective measures of time perception. We hypothesized that participants would make more "long" responses in the hands-near conditions, which would be indicative of a sense of time slowing down.

\section{Method}

Participants After removing three early participants due to equipment malfunction, the remaining sample consisted of 25 undergraduate students (13 males, 12 females), 18 to 28 years of age $(M=21.08, S D=2.53)$, enrolled in a psychology course at Central Michigan University. The sample size was based on studies using similar time perception methodologies (e.g., Gil \& Droit-Volet, 2011) and approximates the sample sizes commonly seen in the peri-hand space literature (e.g., Reed, Grubb, \& Steele, 2006). All participants were recruited through the department's online participant pool and were compensated with course credit. Before data collection, all participants provided informed consent, and the study was approved by the Central Michigan University Institutional Review Board.

Materials and procedure The experiment was run using the EPrime 2.0 (Psychology Software Tools, Pittsburgh, PA) software on a $60-\mathrm{Hz}, 20$-in. computer monitor situated $53 \mathrm{~cm}$ from the participant. Participants completed a temporal bisection task, the parameters of which were based on Gil and Droit-Volet (2011), who had examined the effect of emotion on time perception. The task was divided into three phases: familiarization, practice, and test (Fig. 1a). First, during the familiarization phase, participants were repeatedly shown two stimuli of either a short or a long duration. This was done to give the participants anchors to base their future responses on. Then, during the practice and test phases, participants were shown stimuli with durations between the two anchors. Their task was to identify whether the presented stimuli were closer to the short duration they had previously learned or the long duration.

During the familiarization phase, each trial began with the word "Ready?," centered in gray Calibri font on a black background. After the participants had verbally responded "ready," the experimenter pressed the response button. After a 500-ms interval, participants were presented with a horizontally oriented gray oval $\left(17.04^{\circ} \times 12.99^{\circ}\right.$ of visual angle $)$ on a black background for either $200 \mathrm{~ms}$ (short duration) or $1,800 \mathrm{~ms}$ (long duration). After each presentation, the question "Short or Long?" appeared on the screen, which cued the experimenter to respond "short" or "long" to indicate to the participant which duration was shown. The question remained on the screen until the experimenter had responded and then pressed the response button to move on to the next trial. Each of the two durations was presented five times with participants' hands near the screen (i.e., "hands-near block") and five times with their hands in their laps (i.e., "hands-far block"), for a total of 20 trials during the familiarization phase. The order of the two blocks in this phase was randomly determined, as was the order of stimulus durations for each trial in each block. In the hands-near blocks, participants rested their elbows on the table and aligned their hands with clear tape on the edges of the monitor, such that their fingertips would rest just beyond the horizontal edges of the oval. In the hands-far blocks, participants simply rested their hands in their laps, such that the hands were approximately the same distance from one another as in the hands-near blocks.

Next, participants began the practice phase. The procedure was identical to that in the familiarization phase, except that 


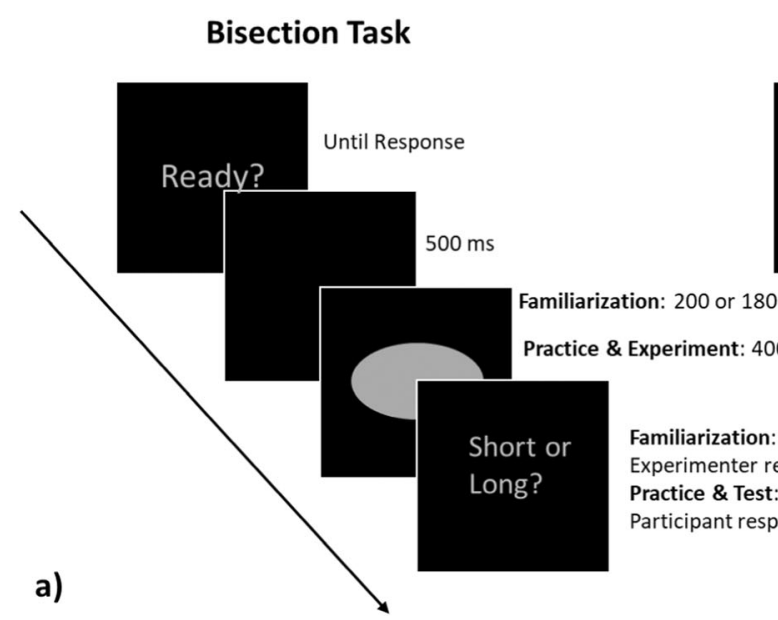

Fig. 1 In the bisection task (a), participants were familiarized with a short and a long anchor duration and then asked to judge which anchor a presented stimulus's duration was closest to. In the verbal estimation

participants were presented with ovals that had one of seven possible durations between 200 and 1,800 ms (e.g., 400, 600, $800,1,000,1,200,1,400$, or $1,600 \mathrm{~ms}$ ), and here they responded instead of the experimenter. Their task was to indicate whether the presented oval was closer to the "short" duration (i.e., $200 \mathrm{~ms}$ ) they had previously learned or the "long" duration (i.e., 1,800 ms), by verbally responding "short" or "long" after each trial. They were told that the actual stimulus duration would be somewhere between the two durations they had previously learned, and to give their best judgment as to which it was closer to. After seven practice trials in a hands-near block and seven practice trials in a hands-far block (i.e., one trial for each duration, with duration and block order randomly determined), they began the test phase.

The test phase was identical to the practice phase, except than it was broken into four 35-trial blocks. Each of the seven durations was randomly presented five times in each block. There were two hands-near and two hands-far blocks, the order of which was randomized, with the beginning posture randomly determined. In-between blocks, participants were told they could take a break if they wanted. Participants were debriefed after the experiment was over, and the study lasted approximately $20-25 \mathrm{~min}$.

\section{Results}

The proportion of "short" responses was analyzed with a 2 (hand distance: near, far) $\times 7$ (duration [in ms]: 400, 600, 800, $1,000,1,200,1,400,1,600)$ repeated measures analysis of variance (ANOVA). Since the assumption of sphericity was violated, a Greenhouse-Geisser correction was applied. As we expected, the main effect of duration was significant, $F(2.94$, $24)=412.14, p<.001, \eta^{2}=.945$, such that participants made fewer "short" responses as duration increased (Fig. 2).
Verbal Estimation Task

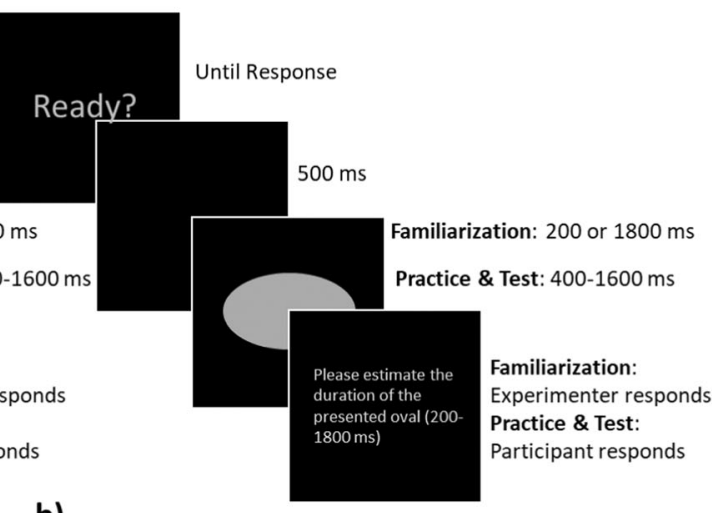

b)

task (b), they were first familiarized with two durations (i.e., 200 and $1,800 \mathrm{~ms}$ ) and then asked to estimate, in milliseconds, for how long the stimuli had been presented

Unexpectedly, the main effect of hand distance was nonsignificant, $F(1,24)=1.35, p=.256, \eta^{2}=.053$, as was the Duration $\times$ Hand Distance interaction, $F(3.05,24)=0.62, p$ $=.716, \eta^{2}=.025$ (Fig. 2). Thus, our hypothesis that participants would experience a slowing down of time in peri-hand space was not supported.

\section{Experiment 2}

In Experiment 2, participants completed a verbal estimation task, which yields explicit measures of the perception of temporal duration. Here we expected participants to overestimate the duration of stimuli in the hands-near conditions, which would be indicative of a sense of time slowing down.

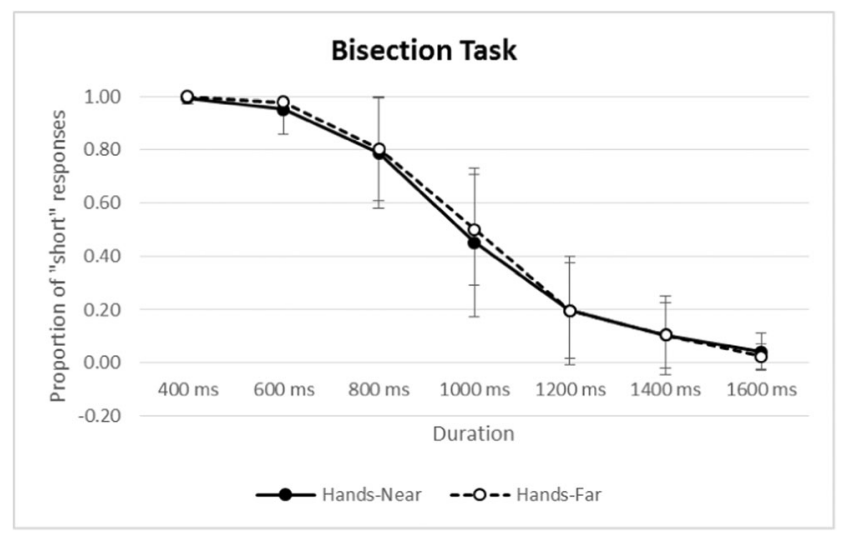

Fig. 2 Proportions of "short" responses as a function of hand distance and duration. The main effect of hand distance and the Hand Distance $\times$ Duration interaction were nonsignificant. Thus, the results suggest that peri-hand space did not affect the temporal perception of the tested durations. Error bars represent one within-subjects standard error of the mean 


\section{Method}

Participants After removing three participants for the same equipment malfunction encountered in Experiment 1, and one for poor performance (i.e., $>2 S D$ s from the grand mean), our sample consisted of 25 new undergraduate students (21 females, four males) 18 to 23 years of age ( $M=$ $20.08, S D=1.26$ ), enrolled in a psychology course at Central Michigan University. All participants were recruited through the department's online participant pool and were compensated with course credit. Before data collection, all participants provided informed consent and the study was approved by the Central Michigan University Institutional Review Board.

Materials and procedure All details of the materials and procedure for Experiment 2 were identical to those of Experiment 1 , with the following exceptions. First, instead of being told they were being shown "short" or "long" stimuli during the familiarization phase, participants were instead given the actual durations, and the experimenter responded "200 ms" or " $1,800 \mathrm{~ms}$," respectively, to a short or a long stimulus. Then, during the practice and test phases, instead of responding "short" or "long," participants were asked to give an explicit temporal judgment, in milliseconds, of how long they estimated a stimulus had been shown for (Fig. 1b).

\section{Results}

A temporal estimation index (TEI) is typically calculated with the verbal estimation task (Gil \& Droit-Volet, 2011), by subtracting the actual duration from the reported duration and then dividing by the actual duration. Therefore, as scores deviate from zero, temporal judgments become less accurate, with negative scores reflecting underestimation and positive scores reflecting overestimation. TEI scores were analyzed in a 2 (hand distance: near, far) $\times 7$ (duration [in ms]: 400, 600, $800,1,000,1,200,1,400,1,600)$ repeated measures ANOVA. The main effect of duration was significant, $F(6,24)=4.99, p$ $=.02, \eta^{2}=.165$. A Bonferroni-corrected post-hoc pairwise comparison showed that participants underestimated the duration of the $800-\mathrm{ms}$ ovals $(M=-0.32, S E=0.04)$ as compared to the 1,400 -ms ovals $(M=-0.18, S E=0.04), t(25)=-2.71, p$ $=.012$; participants also underestimated the duration of the 1,000 -ms ovals $(M=-0.27, S E=0.03)$ as compared to the 1,400-ms ovals, $t(25)=-2.82, p=.009$ (see Fig. 3). This pattern is consistent with the findings of Gil and Droit-Volet (2011), who similarly found that participants underestimated across all durations and that this effect generally attenuated as duration increased. The main effect of hand distance was nonsignificant, $F(1,24)=0.33, p=.57, \eta^{2}=.013$, as was the Duration $\times$ Hand Distance interaction, $F(6,24)=1.21, p=$ $.30, \eta^{2}=.046$. Thus, our hypothesis that participants would experience a slowing down of time in peri-hand space was again not supported.

\section{Discussion}

To review, previous research has noted an array of cognitive changes in peri-hand space, including enhanced temporal acuity. Yet it is unclear whether the hands affect the subjective experience of time. To this end, we tested the effects of perihand space on time perception across two experiments utilizing different time perception paradigms: a bisection task (Exp. 1) and a verbal estimation task (Exp. 2). We hypothesized that the participants in both experiments would overestimate the stimulus durations in the hands-near as compared to the hands-far condition, leading to a higher proportion of "long" responses on the bisection task and longer estimates of duration in the verbal estimation task. Both results would indicate a sense of time slowing down in peri-hand space. However, contrary to our hypotheses, hand distance had no effect on participants' perceptions of time across the tested durations in either experiment.

One explanation for the lack of hand-related differences is that changes in time perception in peri-hand space might manifest at timescales beyond those of our tested durations. Indeed, in a study that showed enhanced detection of temporal gaps in peri-hand space, the researchers utilized gaps of 10-30 ms (Gozli et al., 2012). Similarly, Thomas (2015) found enhanced temporal acuity in peri-hand space in a motion detection task using 50-ms durations. The cognitive and neural mechanisms underlying rapid temporal processing might differ slightly from those involved in relatively longer processing. Indeed, it seems that the cerebellum is the primary structure involved in brief temporal processing, whereas the prefrontal cortex, substantia nigra, and striatum are implicated in suprasecond timing (Koch, Oliveri, \& Caltagirone, 2009). Therefore, it is possible that testing with shorter durations might reveal changes in time perception that our tasks were not sensitive to.

Another explanation for our null results could be that some of the perceptual and attentional changes associated with perihand space are eradicable. Peri-hand effects have been shown to be fragile and sensitive to small changes in task procedure (Andringa, Boot, Roque, \& Ponnaluri, 2018). For instance, Andringa and colleagues were unable to replicate several previously demonstrated effects of peri-hand space on visual attention, such as slowed visual search rate. At first assuming that their disparate results were due to the use of a tablet rather than the typical desktop computer, the authors ran a follow-up study attempting to identically replicate the previous findings, but again they were unable to do so. Since these wellestablished effects had been replicated elsewhere, their study illustrates that peri-hand effects can be affected by small 


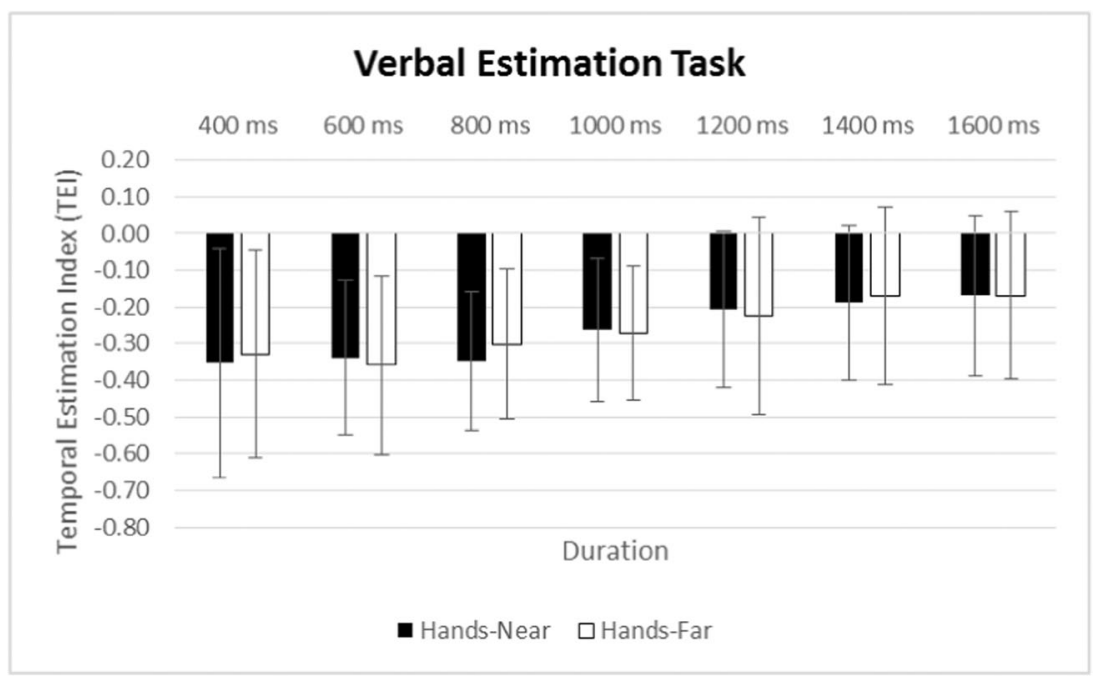

Fig. 3 Verbal estimations of stimulus duration as a function of hand distance and actual stimulus duration. The main effect of duration was significant, such that 800 - and 1,000-ms ovals were underestimated more than 1,400-ms ovals. However, hand distance did not have an effect. Error bars represent one within-subjects standard error of the mean changes in procedure. In fact, even minor changes in grasp posture can lead to opposing effects in visual processing in peri-hand space (Thomas, 2015). Therefore, just as the attentional and perceptual changes in peri-hand space do not manifest uniformly, temporal changes in peri-hand space may be sensitive to seemingly negligible task-level variations.

What else could explain our null results? One possibility involves our assumption that the magnocellular pathway would automatically be engaged when the participants in the present study positioned their hands near the stimuli. Recent research has uncovered instances in which stimuli near the hands are processed through the parvocellular pathway, indicating that the magnocellular pathway is not irrevocably engaged near the hands (Goodhew \& Clarke, 2016). Goodhew and Clark designed a visual search task with stimuli that were either magnocellular-preferred (i.e., low spatial frequency) or parvocellular-preferred (i.e., high spatial frequency) and found that when using large set sizes, participants showed a parvocellular bias in peri-hand space. If the magnocellular system is not engaged in peri-hand space, we would not expect temporal distortions to occur. However, given that the stimuli utilized in our study were gray and had low spatial frequency, the results from Goodhew and Clarke would predict engagement of the magnocellular pathway.

Serendipitously, following data collection, the authors of this article were unexpectedly presented with research from an independent laboratory also investigating the effects of peri-hand space on time perception. Qi, Wang, He, and Du (2019) examined the effect of peri-hand space on temporal reproduction across 1,200-, 1,600-, 2,000-, and 2,800-ms durations. When stimuli were presented near both hands, they found that participants overestimated their reproductions and that this effect remained, although it was attenuated, when only one hand was near the screen. Importantly, the overestimations in peri-hand space were found only at the $1,200-\mathrm{ms}$ duration. This finding supports our assertion that the timescale used in our experiments should be appropriate to detect perihand effects.

Several factors might have led to the disparate results. One explanation is that the tasks might tap into slightly different aspects of temporal perception. Indeed, when Gil and DroitVolet (2011) examined the effects of emotion on time perception using bisection, verbal estimation, and reproduction tasks, they found that emotion exerted unequal effects across the different measures. Further research will be needed to delineate what makes the tasks sensitive to specific temporal distortions. An intuitive explanation for peri-hand effects being shown in a reproduction task, but not in a bisection or verbal estimation task, is that participants responded with their hands in the reproduction task but responded verbally in our bisection and verbal estimation tasks. Since the cognitive changes in peri-hand space are thought to arise to facilitate action with stimuli near the hands, it is conceivable that the attentional and perceptual enhancements would be erased when participants did not need to perform any actions with their hands. However, previous research has revealed peri-hand effects across a variety of tasks and response modalities (Abrams et al., 2008). Taking these findings into account, it would be surprising if the sole reason for our null results were the action taken (or not taken) by the hands. Therefore, the most parsimonious explanation for the conflicting results likely relates to the fragility of peri-hand effects (Andringa et al., 2018; De Grave, Brenner, \& Smeets, 2011) and/or the tasks tapping into different aspects of temporal perception (Gil \& Droit-Volet, 2011). 
Although there is still much to learn about the nuances of temporal processing in peri-hand space, these results lay a foundation for a new line of research regarding the hands' effects on time perception. Despite robust theoretical evidence predicting slowed time processing in peri-hand space, we found across two different experiments that time perception did not change when stimuli were presented near the hands. Interestingly, an independent laboratory concurrently found contradicting evidence when using a temporal reproduction task. This adds to a growing consensus that changes in cognition in peri-hand space are nuanced and sensitive to small task-level variations.

Author note None of the data or materials for the experiments reported here are available, and none of the experiments were preregistered. The authors declare that they have no conflicts of interest. We thank Leah Ryal for her assistance in the data collection.

\section{References}

Abrams, R. A., Davoli, C. C., Du, F., Knapp, W. H., III, \& Paull, D. (2008). Altered vision near the hands. Cognition, 107, 1035-1047. https://doi.org/10.1016/j.cognition.2007.09.006

Abrams, R. A., \& Weidler, B. J. (2014). Trade-offs in visual processing for stimuli near the hands. Attention, Perception, \& Psychophysics, 76, 383-390.

Andringa, R., Boot, W. R., Roque, N. A., \& Ponnaluri, S. (2018). Hand proximity effects are fragile: A useful null result. Cognitive Research: Principles and Implications, 3, 7:1-12. https://doi.org/ 10.1186/s41235-018-0094-7

Davoli, C. C. (2010). The relationship between hand-nearness, emotional arousal, and visual processing (Unpublished doctoral dissertation). Washington University in St. Louis, St. Louis, MO.

De Grave, D. D., Brenner, E., \& Smeets, J. B. (2011). Using a stick does not necessarily alter judged distances or reachability. PLOS ONE, 6 , e16697. https://doi.org/10.1371/journal.pone.0016697

Droit-Volet, S., Fayolle, S., Lamotte, M., \& Gil, S. (2013). Time, emotion and the embodiment of timing. Timing and Time Perception, 1, 99126.

Droit-Volet, S., \& Meck, W. H. (2007). How emotions colour our perception of time. Trends in Cognitive Sciences, 11, 504-513. https://doi. org/10.1016/j.tics.2007.09.008
Du, F., Wang, X., Abrams, R. A., \& Zhang, K. (2017). Emotional processing is enhanced in peri-hand space. Cognition, 165, 39-44.

Gil, S., \& Droit-Volet, S. (2011). "Time flies in the presence of angry faces" ... depending on the temporal task used! Acta Psychologica, 136, 354-362.

Goodhew, S. C., \& Clarke, R. (2016). Contributions of parvocellular and magnocellular pathways to visual perception near the hands are not fixed, but can be dynamically altered. Psychonomic Bulletin \& Review, 23, 156-162. https://doi.org/10.3758/s13423-015-0844-1

Goodhew, S. C., Gozli, D. G., Ferber, S., Pratt, J. (2013). Reduced temporal fusion in near-hand space. Psychological Science, 24, 891900. https://doi.org/10.1177/0956797612463402

Gozli, D. G., West, G. L., \& Pratt, J. (2012). Hand position alters vision by biasing processing through different visual pathways. Cognition, 124, 244-250.

Koch, G., Oliveri, M., \& Caltagirone, C. (2009). Neural networks engaged in milliseconds and seconds time processing: Evidence from transcranial magnetic stimulation and patients with cortical or subcortical dysfunction. Philosophical Transactions of the Royal Society B, 364, 1907-1918. https://doi.org/10.1098/rstb.2009.0018

Kobayashi, M., \& Ichikawa, M. (2016). Emotions evoked by viewing pictures may affect temporal aspects of visual processing. Japanese Psychological Research, 58(3), 273-283.

Qi, Y., Wang, X., He, X., \& Du, F. (2019). Prolonged subjective duration near the hands: Effects of hand proximity on temporal reproduction. Manuscript under review.

Reed, C. L., Grubb, J. D., \& Steele, C. (2006). Hands up: Attentional prioritization of space near the hand. Journal of Experimental Psychology: Human Perception and Performance, 32, 166-177. https://doi.org/10.1037/0096-1523.32.1.166

Schendel, K., \& Robertson, L. C. (2004). Reaching out to see: Arm position can attenuate human visual loss. Journal of Cognitive Neuroscience, 16, 935-943.

Stetson, C., Fiesta, M. P., \& Eagleman, D. M. (2007). Does time really slow down during a frightening event? PLoS ONE, 2, e1295. https:// doi.org/10.1371/journal.pone.0001295

Thomas, L. E. (2015). Grasp posture alters visual processing biases near the hands. Psychological Science, 26, 625-632.

Watson, A. B. (1986). Temporal sensitivity. In Handbook of perception and human performance (1st ed., pp. 6-1 to 6-43). NY: WileyInterscience.

Tipples, J. (2008). Negative emotionality influences the effects of emotion on time perception. Emotion, 8, 127-131.

Publisher's note Springer Nature remains neutral with regard to jurisdictional claims in published maps and institutional affiliations. 